\title{
Estimation of Carbon Content in the Páramo Ecosystem of Pasa Population, Ambato Can- ton, Tungurahua Province
}

\section{Estimación de Carbono Almacenado en el Páramo de la Parroquia Pasa, Cantón Ambato, Provincia De Tungurahua}

\author{
M. Guallpa, V. Espinoza, and D. Arcos
}

Memories II International

Congress Forests and

Agroforestry for the 21st

Century

Corresponding Author:

M. Guallpa

victor.espinoza@espoch.edu.ec

Published: 21 January 2021

Production and Hosting by

Knowledge E

(c) M. Guallpa et al. This article is distributed under the terms of the Creative Commons Attribution License, which permits unrestricted use and redistribution provided that the original author and source are credited.
S OPEN ACCESS
Escuela Superior Politécnica de Chimborazo, Facultad de Recursos Naturales, Carrera de Ingeniería Forestal Riobamba, Ecuador

\section{Abstract}

The stored carbon content was determined based on a randomized stratified experimental design by type of vegetation; three sampling units of $400 \mathrm{~m}^{2}$ each were installed, from 36004100 m.s.n.m., with intervals of $150 \mathrm{~m}$ of altitude. The inventory of herbaceous vegetation was taken using the GLORIA method. For the sampling of the aerial biomass and necromass in the plot, circular subplots were constructed, and the samples were collected in 4 quadrants of $0.25 \mathrm{~m}^{2}$, two in each subplot. Samples of 0-20, 20-40 and 40-60 cm were taken from the soil profile, and the percentage of organic carbon in the soil and the apparent density were evaluated. The results indicate a floristic diversity represented by 44 species, 39 of herbaceous vegetation and 5 of tree vegetation. The carbon content stored in aerial biomass returned a value of $83.88 \mathrm{tC} \cdot \mathrm{ha}^{-1}$ for herbaceous vegetation and $158.50 \mathrm{tC} \cdot \mathrm{ha}^{-1}$ in tree vegetation, and in terms of necromass, returned values of $0.89 \mathrm{tC}^{-h a^{-1}}$ and $7.40 \mathrm{tC}-\mathrm{ha}^{-1}$, respectively. The carbon stored in the soil at a depth of $20 \mathrm{~cm}$ is $111.84 \mathrm{tC}-\mathrm{ha}^{-1}$ for herbaceous vegetation and $122.45 \mathrm{tC}^{-\mathrm{ha}^{-1}}$ for tree vegetation.

Keywords: ecosystem, paramo, tree vegetation, carbon, biomass.

\section{Resumen}

Se determinó el contenido de carbono almacenado en base a un diseño experimental estratificado al azar por tipo de vegetación, se instalaron tres unidades de muestreo de 400 $\mathrm{m}^{2}$ cada una, a partir de 3600-4100 m.s.n.m; con intervalos de $150 \mathrm{~m}$ de altitud. El inventario de la vegetación herbácea mediante el método GLORIA, Para el muestreo de la biomasa aérea y necromasa en la parcela se anidaron subparcelas circulares, se recolecto las muestras en 4 cuadrantes de $0.25 \mathrm{~m}^{2}$ dos en cada subparcela. En el comportamiento suelo se tomaron muestras de $0-20 \mathrm{~cm}, 20-40 \mathrm{~cm}$ y de $40-60 \mathrm{~cm}$, se evaluó el porcentaje de carbono orgánico en el suelo y la densidad aparente. Los resultados indican una diversidad florística representada por 44 especies, 39 de la vegetación herbácea y 5 de la vegetación arbórea. El contenido de carbono almacenado en la biomasa aérea reporto un valor de $83,88 \mathrm{tC} \cdot \mathrm{ha}^{-1}$ para vegetación herbácea y de 158,50 tC.ha ${ }^{-1}$ en la vegetación arbórea, en cuanto a necromasa con valores de $0,89 \mathrm{tC}$ - ha $\mathrm{a}^{-1}$ y de 7,40 tC- ha ${ }^{-1}$ respectivamente. El carbono almacenado en el suelo a 20 $\mathrm{cm}$ de profundidad es de 111.84 tC- ha ${ }^{-1}$ la vegetación herbácea y de 122,45 tC- ha ${ }^{-1}$ para la vegetación arbórea. 


\section{Introducción}

El páramo es el ecosistema más frágil que posee la provincia de Tungurahua, en los últimos tiempos se ha visto afectado principalmente por el cambio del uso del suelo y el avance de la frontera agrícola, pese a su gran importancia en la provisión de servicios hidrológicos y protección de la biodiversidad, en cuanto a su capacidad para almacenar carbono es un tema prácticamente desconocido para la mayoría de personas. Dentro del nuevo modelo de gestión del Honorable Gobierno Provincial de Tungurahua se da la importancia que este ecosistema tiene en cuanto sus servicios ambientales, a través del Plan de Manejo de Páramo Pasa 2018 financiado por Gobierno Provincial de Tungurahua, el Fondo de Manejo de Páramo y Lucha contra la Pobreza, el cual es ejecutado por la Central Ecuatoriana de Servicios Agrícolas, se determinó el contenido de carbono almacenado en la biomasa aérea y en el suelo, con el objetivo de presentar a las comunidades locales y de la provincia la importancia de tal servicio ambiental y la necesidad de multiplicar las acciones para conservar el ecosistema páramo y así evitar el avance de la frontera agrícola y por consiguiente el cambio del uso del suelo, los cuales son las principales causales para la alteración y destrucción de ecosistemas, lo que acelera el cambio climático.

Es imperativo que las comunidades locales, principales actores de la conservación del páramo reciban beneficios: Económicos, sociales, ambientales. La presente investigación recopiló información de indicadores que sustentaran la valoración por conservación ya que resalta varios servicios ambientales que brinda el ecosistema páramo, tales como: Protección de fuentes hídricas, aumento en la cantidad y calidad de agua, conservación de especies de flora y fauna y además el secuestro y almacenamiento de carbono que se puede negociar como medida para reducir las emisiones de gases de efecto invernadero asociados a deforestación y degradación forestal.

Al conocer valores reales en cuanto al almacenamiento de carbono en los diferentes sumideros, se plantearán proyectos que contribuyan a la mitigación del cambio climático, los mismos se podrán trabajar con las comunidades y las instituciones que se encuentran dentro del convenio del Plan de Manejo de Páramos Pasa.

\section{Materiales y Métodos}

\subsection{Delimitación del área en estudio}

La parroquia Pasa geográficamente se encuentra ubicada en el sector occidental de Tungurahua, a $17 \mathrm{~km}$ de Ambato con una extensión total de 4884 ha. De las cuales 1987 ha están formadas por bosque nativo y páramo herbáceo. Ecosistemas manejados bajo un esquema de conservación. Al existir los dos tipos de vegetación fueron seleccionados para la estimación de contenido de carbono. 


\subsection{Características específicas del campo experimental}

El presente estudio se realizó en la vegetación herbácea y arbórea de 4 pisos altitudinales en el páramo de la parroquia Pasa. La fase de campo se inició el 16 de agosto del 2018 y culmino el 21 de febrero del 2019. Para la ejecución de la investigación se tomaron los datos en 12 parcelas de la vegetación herbácea y 6 parcelas en la vegetación arbórea, en este caso fueron 6 ya que no existe vegetación arbórea en la altitud 1 y altitud 2. Las especificaciones del campo experimental fueron:

Número de tratamientos: 18 con 3 repeticiones

Número de unidades experimentales: 53

\subsection{Establecimiento de parcelas para el muestreo}

En la vegetación herbácea se instalaron 12 parcelas y en la vegetación arbórea 6 parcelas con unidades de muestreo permanentes de forma cuadrada con la dimensión de $20 \times 20 \mathrm{~m}$ en base a la metodología propuesto por CONDESAN (2013), con una modificación en el tamaño de las parcelas.

\subsection{Inventario de la vegetación}

Para el inventario de la vegetación herbácea en la parcela general de $400 \mathrm{~m}^{2}$ se aplicó parte de la metodología GLORIA (Iniciativa para la Investigación y el Seguimiento Global de los Ambientes Alpinos [2], mediante la instalación de una unidad de muestreo anidada de $25 \mathrm{~m}^{2}$ por piso altitudinal en el centro de cada parcela. Para su establecimiento se ubicó el punto de inicio (Subparcela 1) a partir de ahí se midió 5 m horizontales con un azimut de $0^{\circ}$ (norte franco) ubicando el punto 2 (Subparcela 2), luego con un azimut de $180^{\circ}$ (Este franco) y a $5 \mathrm{~m}$ de distancia se colocó el punto 3 (Subparcela 3), y finalmente a una distancia de $5 \mathrm{~m}$ y con un azimut de $180^{\circ}$ (sur franco) se instaló el punto 4 (Subparcela 4). En cada uno de los vértices de la parcela de $25 \mathrm{~m}^{2}$ se ubicó la subparcela de $1 \mathrm{~m}^{2}$, hecha con un armazón de madera y con celdas de 0,01 $\mathrm{m}^{2}$, en forma de malla, cada celda determina el $1 \%$ del cuadrante. En el bosque nativo se registró las siguientes variables: Altura, diámetro a la altura del pecho (DAP). De cada especie se tomaron 2 muestras botánicas.

\subsection{Estimación de carbono de la necromasa de la vegetación her- bácea}

En la parcela general de $20 \times 20 \mathrm{~m}$ se anidaron subparcelas circulares con un radio de 10 y $5 \mathrm{~m}$, se recolecto la necromasa en 4 cuadrantes de $0,25 \mathrm{~m}^{2}$, dos en la subparcela circular de $10 \mathrm{~m}$ radio y dos en la subparcela de $5 \mathrm{~m}$ de radio, en forma de cruz. Las muestras de necromasa recolectadas, se pesó y se llevó al laboratorio para su secado (usualmente entre 48 y $72 \mathrm{hr}$ ) a $70^{\circ} \mathrm{C}$ [3]. Para la estimación de contenido de carbono en la necromasa se multiplico el valor de necromasa seca por 0,5 como indica el IPCC [4]. 


\subsection{Estimación de carbono en la biomasa de la vegetación her- bácea}

En la parcela general de $20 \times 20 \mathrm{~m}$ se anidaron dos parcelas circulares con un radio de 10 y $5 \mathrm{~m}$. En cada parcela circular se cosecho la biomasa en 4 cuadrantes o sub unidades de muestreo de $0,25 \mathrm{~m}^{2}$ dos en la subparcela circular de $10 \mathrm{~m}$ de radio y dos en la de $5 \mathrm{~m}$ de radio, en forma de cruz. En las cuatro subunidades de muestreo de 0,25 $\mathrm{m}^{2}$, se cosecho toda la vegetación presente, cortándola al ras del suelo con una tijera podadora. Las muestras colectadas fueron pesadas, empacadas y se las traslado al laboratorio para determinar el contenido de carbono. Las muestras de biomasa fueron secadas a $70^{\circ} \mathrm{C}$ entre 48 a $72 \mathrm{hr}$ hasta alcanzar su peso seco. Para estimar el contenido de carbono por subparcela se calculó el contenido de humedad (Ecuación 1).

$$
C H=\frac{P V S-P S S}{P V S},
$$

dónde: $\mathrm{CH}$ es el contenido de humedad; PVS es el peso verde de la submuestra (g) y PSS es el peso seco de la submuestra (g).

Para el cálculo de carbono por subparcela se lo realizó con la Ecuación 2.

$$
Y=P V T-(P V T \times C H),
$$

dónde: Y es la biomasa por subparcela y compartimiento (g); PVT es el peso húmedo total de la muestra (g) y $\mathrm{CH}$ el contenido de humedad de la submuestra. Para convertir los datos de biomasa a cantidad de carbono, se multiplicó el valor de biomasa por 0,5 como indica el IPCC [4].

\subsection{Estimación de carbono del suelo en la vegetación herbácea}

En las 12 unidades de muestreo de $20 \times 20 \mathrm{~m}$, con el barreno se procedió a la toma de 5 submuestras a tres profundidades: $0-20 \mathrm{~cm}, 20-40 \mathrm{~cm}$ y de $40-60 \mathrm{~cm}$, las submuestras se tomaron en cada esquina de la parcela y una en el centro. Las submuestras se mezclaron formando una muestra compuesta por cada profundidad. Las muestras se empacaron herméticamente y se etiquetaron con el número de parcela, la vegetación, la profundidad, altitud, y responsable del muestreo.

La características físicas y químicas de las muestras se realizaron con los siguientes métodos: Porcentaje de materia orgánica (ignición), \% de carbono orgánico en el suelo (Walldey \& Black), densidad aparente $\mathrm{g} / \mathrm{cm}^{3}$ (gravimétrico-cilindro), densidad real $\mathrm{g} / \mathrm{cm}^{3}$ (Gravimétrico-picnómetro), textura (Bouyoucus), $\mathrm{pH}$ (electroquímico), conductividad eléctrica (electroquímico), nitrógeno en ppm (Dumas), fósforo en ppm (UVVIS), potasio (meq/100 g), calcio (meq/100), magnesio (meq/100), Cobre en (meq/100), manganeso (meq/100), zinc en (meq/100). 


\subsection{Estimación de carbono en el bosque nativo}

La estimación de contenido de carbono en la biomasa de la vegetación arbórea, se realizó por el método indirecto con la siguiente ecuación general propuesta por Calderon y Lozada [5]

$$
B A=V C * D * F E B,
$$

donde: BA: Biomasa aérea (ton/ha); VC: Volumen comercial ( $\mathrm{m}^{3}$ ); V: AB*H*FF; AB: (DAP)2 ${ }^{*} \pi / 4$; D: Densidad básica de la madera $\left(t / \mathrm{m}^{3}\right)$; FEB: Factor de expansión de la biomasa.

\subsection{Estimación de carbono de la necromasa en el bosque nativo}

En la parcela general de $20 \times 20$ m instaladas en la vegetación arbórea, se anidaron subparcelas circulares con un radio de 10 y $5 \mathrm{~m}$, se recolecto la necromasa en 4 cuadrantes de $0,25 \mathrm{~m}^{2}$, dos en la subparcela circular de $10 \mathrm{~m}$ radio y dos en la subparcela de $5 \mathrm{~m}$ de radio, en forma de cruz. Las muestras de necromasa recolectadas fueron: Hojarasca, ramas caídas, individuos muertos en pie, se pesó y se llevó al laboratorio para su secado a $70^{\circ} \mathrm{C}[3]$

\subsection{Estimación de carbono del suelo en el estrato arbóreo}

En las 6 parcelas permanentes de $20 \times 20$ m instaladas en el estrato arbóreo se procedió a la toma de 5 submuestras, una en cada esquina de la parcela y una en el centro. Con el barreno se tomaron muestras a tres profundidades, $0-20 \mathrm{~cm}, 20-40 \mathrm{~cm}$ y de $40-60 \mathrm{~cm}$, cada una de las submuestras se mezcló formando una muestra compuesta por cada profundidad. La características físicas y químicas de las muestras se determinaron por los siguientes métodos: Porcentaje de materia orgánica (ignición), \% de carbono orgánico en el suelo (Walldey \& Black), densidad aparente $\mathrm{g} / \mathrm{cm}^{3}$ (gravimétrico-cilindro), densidad real $\mathrm{g} / \mathrm{cm}^{3}$ (Gravimétrico-picnómetro), textura (Bouyoucus), $\mathrm{pH}$ (electroquímico), conductividad eléctrica (electroquímico), nitrógeno en ppm (Dumas), fósforo en ppm (UV-VIS), potasio (meq/100 g), calcio meq/100, magnesio meq/100, cobre en meq/100, manganeso meq/100, zinc en meq/100.

\section{Resultados y Discusión}

\subsection{Inventario en la vegetación herbácea}

En la composición florística del páramo herbáceo de 3600-4200 m.s.n.m. la familia dominante es Asteraceae con la mayor diversidad relativa del 25,64\%, Poaceae con el 12,82\%, y las familias menos abundantes: Alstromeriaceae, Blechnaceae, Caryophyllaceae, Cyperacea, Ericaceae, Geraneaceae, Lycopodiaceae, Polygalacea, Polygonaceae, Ranunculacea con un valor similar de $2,56 \%$ para cada una de ellas.

Los resultados a nivel de familias predominantes en esta investigación concuerdan con lo determinado por Pujos [6] quien reportó a las familias; Asteraceae, Poaceae 
y Apiaceae como las más diversas en el páramo de las organizaciones del pueblo Chibuleo, provincia de Tungurahua con similar método de muestreo.

\subsection{1. Índice de Shannon-Weaver}

Según el índice de Shannon-Weaver la diversidad es media al encontrarse entre los valores 1,36-3,5 en los 4 pisos altitudinales evaluados, siendo el piso altitudinal 3 el que mayor diversidad presento con 3,13 y el valor más bajo 2,09 que corresponde al piso altitudinal 4 (Tabla 1). El índice promedio de Shannon-Weaver con un valor de 2,58 para una altitud de 3600-3750 m.s.n.m es ligeramente superior al valor de 2,39 determinado en el estudio realizado en el páramo herbáceo de Pasa [7] resultados que confirman la diversidad media que posee el sitio evaluado.

\subsection{2. Índice de Simpson}

El índice de Simpson fluctúa de 0,63-0,84 lo que representa la diversidad es de media a alta. Los pisos altitudinales que presentan diversidad media son los pisos 1 y 4 . Mientras que los pisos 2 y 3 presentan diversidad alta. El piso altitudinal 4 es el que presenta un menor valor con un 0,63 mientras que el piso altitudinal 3 es el que presenta el mayor valor con un 0,84 (Tabla 2). El índice promedio de Simpson con un valor de 0,75 para el estrato herbáceo es ligeramente menor al determinado en el estudio realizado en el páramo herbáceo de Pasa [7].

\subsection{3. Índice de Jaccard}

Según el índice de similitud de Jaccard en el muestreo presenta alta similitud entre la parcela 2 y la parcela 3 en un rango altitudinal de 3750-3900 y 3900-4050 m.s.n.m., respectivamente con un valor del $88 \%$ y la menor similitud se presentó entre las parcelas 1 y 4 con el $15 \%$ de similitud.

\subsection{Inventario de la vegetación arbórea}

\subsection{1. Índice de valor de importancia de las familias y especies arbóreas del bosque nativo}

En la composición florística del bosque nativo de 3750-4050 m.s.n.m. la familia dominante es Rosaceae con la mayor diversidad relativa del $46,86 \%$, al registrar el mayor número de individuos mientras que la familia con el menor índice es Scrophulariaceae con el menor número de individuos y una sola especie con un valor de $8,31 \%$. Los resultados obtenidos a nivel de familias predominantes a nivel de la vegetación arbórea en esta investigación son similares al comparar con el 61,33\% de la familia Rosaceae y el 38,66\% para la familia Asteraceae de acuerdo al estudio del Bosque Sachafilo de la parroquia desarrollado por Londo [7]. 
En cuanto al índice de valor de importancia de acuerdo a las especies registradas dentro del estrato arbóreo (Tabla 3), la especie Polylepis reticulata (Rosaceae) presenta el mayor índice de diversidad con un $88,74 \%$ siendo la especie de mayor valor dentro de nuestro estudio para este tipo de vegetación evaluada, seguida de la especie Gynoxis buxifolia (Kunth) Cass (Asteraceae) con un I.V.I. de 10,76\% y finalmente con el menor índice de valor de importancia fue Gynoxys fuliginosa (Kunth) Cass (Asteraceae). Resultados que difieren con el estudio desarrollado a similar altitud localizado a una distancia de $2 \mathrm{~km}$, donde se reportó a las especies con el mayor I.V.l; Polylepis incana y Gynoxis halli Hieron según Londo [7].

Las especies diagnosticadas según MAE [8] son: Buddleja incana, B. pichinchensis, Columellia oblonga, Escallonia myrtilloides, Geranium ayavacense, Gynoxys acostae, G. cuicochensis, G. hallii, Hesperomeles ferruginea, H. obtusifolia, Luzula gigantea, Polylepis incana, P. lanuginosa, P. microphylla, P. pauta, P. reticulata, P. sericea, P. weberbaueri, Rubus coriaceus, de las cuales: Polylepis reticulata, Buddleja incana, Gynoxys sp coinciden con los resultados de esta investigación de forma parcial.

\subsection{2. Índice de Shannon-Weaver}

El índice de diversidad de Shannon-Weaver calculado de las especies arbóreas es de 0,80 (Tabla 4), Según la interpretación de [9], la vegetación arbórea presenta una comunidad forestal diversa. Este resultado concuerda con el de Londo [7] en el que el índice de Shannon-Weaver fue de 0,79 encontrándose en el mismo rango de comunidad forestal diversa. Cuyo estudio fue realizado en el bosque Sachafilo, del páramo en conservación de la parroquia Pasa.

\subsection{3. Índice de Simpson}

El índice de diversidad de Simpson calculado de las especies arbóreas es de 0,42 (Cuadro 18), Según la interpretación de [9] (Tabla 5), la vegetación arbórea presenta una Diversidad media. Este resultado concuerda con el de Londo [7] en el que el IDS fue de 0,53 encontrándose en el mismo rango de diversidad media.

\subsection{Análisis del contenido del carbono}

\subsubsection{Análisis de varianza del $t C \cdot h a^{-1}$ almacenado en la biomasa aérea}

Según el análisis de varianza (Tabla 6) para el carbono orgánico en la biomasa de la vegetación herbácea y arbórea los resultados presentaron que hay diferencias altamente significativas para la variable tratamientos y diferencias significativas en el factor vegetación e interacción. El coeficiente de variación fue de 26,04\% con una, media de 109,30 tC.ha ${ }^{-1}$.

Mediante la prueba de Tukey al 5\% de significancia para el carbono orgánico en la biomasa se estableció que la variable vegetación presenta 2 rangos de significancia (a, b), la vegetación arbórea alcanzo el rango 'a' con una media de 125,79 tC.ha ${ }^{-1}$ 
mientras que la vegetación herbácea alcanzo el rango 'b' con la media más baja de $83,88 \mathrm{tC} \cdot \mathrm{ha}^{-1}$.

En el presente estudio se determinó que la vegetación arbórea es la que mayor cantidad de carbono orgánico almacena con una media de 125,79 tC.ha ${ }^{-1}$, frente a la vegetación herbácea que presenta una media de $83,88 \mathrm{tC} \cdot \mathrm{ha}^{-1}$. Los valores aquí obtenidos guardan concordancia con los valores de los resultados obtenidos por Ayalaet al. [10] en donde se obtuvo un valor medio de 153,86 tC.ha ${ }^{-1}$ en la biomasa del páramo arbustivo; y, 115,30 tC $\cdot \mathrm{ha}^{-1}$ en la biomasa del páramo herbáceo del PNY, la variación de concentración de carbono en la biomasa, es debido al tipo de vegetación mientras más leñosas sean las especies que conforman un estrato, es mayor la capacidad de almacenamiento de carbono orgánico del mismo. Esta tendencia del incremento del almacenamiento de carbono en especies leñosas es corroborada por los resultados obtenidos por Santín y Vidal [11], en los páramos del PNP donde señalan que la biomasa y necromasa de mayor contenido de carbono es la de tipo arbustivo, con un valor de $1,45 \mathrm{~kg} / \mathrm{C} / \mathrm{m}^{2}$ y, en el páramo herbáceo el menor valor es $0,29 \mathrm{~kg} / \mathrm{C} / \mathrm{m}^{2}$.

\subsubsection{Análisis de varianza de $t C \cdot h a^{-1}$ almacenado en la necromasa}

Según el análisis de varianza (Tabla 7) para el carbono orgánico en la necromasa de la vegetación herbácea y arbórea los resultados presentaron diferencias altamente significativas en los tratamientos, altitud, vegetación e interacción y entre los tratamientos vs el resto. El coeficiente de variación fue de 13,34\% con una, media de 3,05 tC.ha ${ }^{-1}$.

Mediante la prueba de Tukey al $5 \%$ de significancia para el carbono orgánico en la necromasa se estableció que la vegetación presenta 2 rangos de significancia (a, b), la vegetación arbórea alcanzo el rango 'a' con una media $7,40 \mathrm{tC} \cdot \mathrm{ha}^{-1}$ mientras que la vegetación herbácea alcanzo el menor rango 'b' con 0,89 tC.ha ${ }^{-1}$. En la interacción entre los testigos y el resto de tratamientos presenta 2 rangos de significancia $(a, b)$, la media más alta con $4,14 \mathrm{tC} \cdot \mathrm{ha}^{-1}$, mientras que la menor fue de $0,86 \mathrm{tC} \cdot \mathrm{ha}^{-1}$.

\subsubsection{Análisis de varianza de $t C \cdot h a^{-1}$ almacenado en el suelo}

Según el análisis de varianza para el carbono orgánico en el suelo de los dos tipos de vegetación los resultados presentaron diferencias significativas entre el testigo y el resto y diferencias altamente significativas entre la altitud inferior y superior. El coeficiente de variación fue de 46,26\% con una, media de 130,27 tC.ha ${ }^{-1}$.

Mediante la prueba de Tukey al 5\% de significancia para el carbono orgánico en el suelo de los dos tipos de vegetación se estableció que en la interacción entre los testigos y el resto de tratamientos presenta 2 rangos de significancia (a, b), la media más alta con el rango 'a' de 156,51 tC.ha ${ }^{-1}$, mientras que el menor fue de 117,15 tc.ha ${ }^{-1}$ con el rango 'b'. En la interacción entre la altitud superior e inferior de tratamientos presenta 2 rangos de significancia (a, b), la media más alta con $200,47 \mathrm{tC} \cdot$ ha $^{-1}$, para el rango 'a', mientras que la menor fue de $112,56 \mathrm{tC} \cdot \mathrm{ha}^{-1}$ para el rango ' $b$ '.

Los resultados obtenidos para la cantidad de carbono orgánico almacenado en la necromasa, presenta una variabilidad con respecto a la altitud. Así en el rango altitudinal 
de 3900-4050 m.s.n.m., se obtuvo una media de $4,96 \mathrm{tC} \cdot \mathrm{ha}^{-1}$, m, mientras que para el rango altitudinal de 3750-3900 m.s.n.m. se determinó una media de 3,33 tC·ha ${ }^{-1}$. Estos resultados guardan concordancia con los expresado por [12, 13].

La tasa de descomposición a mayores altitudes es más lenta en los suelos, debido a la gran humedad sumada a climas fríos, lo que resulta en una capa gruesa de suelo orgánico. En relación a la cobertura vegetal determinó que la vegetación arbórea es la que mayor cantidad de carbono orgánico almacena con una media de 7,40 tC.ha ${ }^{-1}$, frente a la vegetación herbácea que presenta una media de 0,89 tC.ha ${ }^{-1}$. Los datos aquí obtenidos guardan concordancia con los datos obtenidos por Ayala et al. [10] en donde se obtuvo un valor medio de $5,19 \mathrm{tC} \cdot \mathrm{ha}^{-1}$ en la biomasa del páramo arbustivo; y 0,87 tC $\cdot$ ha $^{-1}$ en la biomasa del páramo herbáceo del PNY, la variación de concentración de carbono en la biomasa es debido al tipo de vegetación mientras más leñosas sean las especies que conforman un estrato, es mayor la capacidad de almacenamiento de carbono orgánico del mismo.

\section{Table 1}

Índice de Shannon-Weaver del estrato herbáceo.

\begin{tabular}{|c|c|c|c|}
\hline \multicolumn{4}{|c|}{ Altitud rango altitudinal valor calculado valor referencial interpretación } \\
\hline $13600-3750$ & 2,44 & $1,6-3,0$ & Diversidad media \\
\hline $23750-3900$ & 2,69 & $1,6-3,0$ & Diversidad media \\
\hline $33900-4050$ & 3,13 & $>3,5$ & Diversidad media \\
\hline $44050-4200$ & 2,09 & $1,6-3,0$ & Diversidad media \\
\hline
\end{tabular}

Table 2

Índice de Simpson del estrato herbáceo.

\begin{tabular}{lll|ll} 
& \multicolumn{4}{l}{ Altitud rango altitudinal valor calculado valor referencial interpretación } \\
\hline $\mathbf{1}$ & $3600-3750$ & 0,74 & $0,35-0,75$ & Diversidad media \\
$\mathbf{2}$ & $3750-3900$ & 0,81 & $0,76-1,00$ & Diversidad alta \\
$\mathbf{3}$ & $3900-4050$ & 0,84 & $0,76-1,00$ & Diversidad alta \\
$\mathbf{4}$ & $4050-4200$ & 0,63 & $0,35-0,75$ & Diversidad media
\end{tabular}

\section{Conclusiones}

Según el Índice de Shannon y Simpson la diversidad es alta con un valor de 2,58 y 0,75, respectivamente, la especie dominante es Plantago rígida en la altitud de 3600-3750 m.s.n.m., Calamagrostis intermedia de 3750-3900 m.s.n.m., Azorella pendunculata de 3900-4050 m.s.n.m. y 4050-4200 m.s.n.m. La similitud en cuanto a las especies es mayor entre la altitud $1(3600-3750)$ y la altitud $3(3900-4050)$ y la menor similitud esta entre la altitud 2 (3750-3900) y la altitud 4 (4050-4200) en la vegetación herbácea.

De las especies registradas en el bosque Polylepis reticulata (Rosaceae) presenta el mayor índice de diversidad y con el menor índice de valor de importancia fue Gynoxys 
Table 3

Índice de Valor de Importancia de las especies arbóreas.

\begin{tabular}{|l|l|l|l|l|l|l} 
Familia & $\begin{array}{l}\text { Especie } \\
\text { Rosaceae }\end{array}$ & \# Ind. & AB & Dom R & Den R & IVI \\
\hline $\begin{array}{l}\text { Polylepis } \\
\text { reticulata }\end{array}$ & 407 & 8,14 & 94,01 & 73,47 & 83,74 \\
\hline $\begin{array}{l}\text { Scrophulariaceae } \\
\text { Asteraceae }\end{array}$ & $\begin{array}{l}\text { Budleja incana } \\
\text { Grosvenoria } \\
\text { campii R.M. King } \\
\text { \& H. Rob }\end{array}$ & 19 & 0,26 & 3,02 & 3,43 & 3,22 \\
\hline $\begin{array}{l}\text { Gynoxys buxifolia } \\
\text { (Kunth) Cass }\end{array}$ & 109 & 0,04 & 0,43 & 2,35 & 1,39 \\
\hline $\begin{array}{l}\text { Gynoxys } \\
\text { fuliginosa (Kunth) } \\
\text { Cass }\end{array}$ & 6 & 0,06 & 0,70 & 1,08 & 0,89 \\
\hline
\end{tabular}

Table 4

Índice de Shannon-Weaver del estrato arbóreo.

\begin{tabular}{|c|c|c|c|c|c|}
\hline Familia & Especie & $\#$ & $\mathbf{P i}$ & Log e Pi & $\mathrm{Pi}^{*}(\log \mathrm{e}$ \\
\hline Rosaceae & Polylepis reticulata Kunth & 407 & 0,73 & $-0,31$ & $-0,23$ \\
\hline Scrophulariaceae & Buddleja incana & 19 & 0,03 & $-3,37$ & $-0,12$ \\
\hline Asteraceae & $\begin{array}{l}\text { Grosvenoria campii R.M. } \\
\text { King \& H. Rob }\end{array}$ & 13 & 0,02 & $-3,75$ & $-0,09$ \\
\hline Asteraceae & $\begin{array}{l}\text { Gynoxis buxifolia (Kunth) } \\
\text { Cass }\end{array}$ & 109 & 0,20 & $-1,63$ & $-0,32$ \\
\hline \multirow[t]{2}{*}{ Asteraceae } & $\begin{array}{l}\text { Gynoxys fuliginosa (Kunth) } \\
\text { Cass }\end{array}$ & 6 & 0,01 & $-4,53$ & $-0,05$ \\
\hline & & 554 & & & $-0,80$ \\
\hline
\end{tabular}

Table 5

Índice de Simpson del estrato arbóreo.

Familia
Rosaceae
Scrophulariaceae
Asteraceae
Asteraceae
Asteraceae

Especie
Polylepis reticulata
Buddleja incana
Grosvenoria campii R.M. King \&
H. Rob
Gynoxis buxifolia (Kunth) Cass
Gynoxys fuliginosa (Kunth) Cass

\begin{tabular}{l|l|l|}
$\#$ & $\mathbf{P i}$ & Pi^2 \\
\hline 407,00 & 0,73 & 0,54 \\
19,00 & 0,03 & 0,00 \\
13,00 & 0,02 & 0,00 \\
109,00 & 0,20 & 0,04 \\
6,00 & 0,01 & 0,00 \\
& 554,00 & 0,58
\end{tabular}

fuliginosa (Kunth) Cass (Asterácea), el índice según Shannon-Weaver y Simpson la diversidad es media con un valor de 0,80 y 0,53, respectivamente.

El contenido de carbono en el compartimiento de la biomasa es de 83,88 tC.ha ${ }^{-1}$ en la vegetación herbácea y de 158,50 tC·ha ${ }^{-1}$ para la vegetación arbórea y en la biomasa 
Table 6

Análisis de varianza de $\left(t C \cdot h a^{-1)}\right.$ en la biomasa.

\begin{tabular}{llllllll} 
F. Variación & GI & S. Cuad & C. Medio & Fisher & P. Fisher & Interpretación \\
\hline Total & 17 & $49.069,90$ & & & & \\
\hline Bloques & 2 & 3037,00 & 1518,50 & 1,87 & 0,20 & ns \\
\hline Tratamiento & 5 & $37.931,72$ & 7586,34 & 9,36 & 0,00 & $* *$ \\
\hline Altitud & 1 & 7960,36 & 5093,91 & 6,29 & 0,03 & $*$ \\
\hline Vegetación & 1 & $16.705,18$ & $16.705,18$ & 20,62 & 0,00 & $*$ \\
\hline Interacción & 1 & 7561,63 & 7561,63 & 9,33 & 0,01 & $*$ \\
\hline Ts vs Resto & 1 & 5093,91 & 5093,91 & 6,29 & 0,13 & ns \\
\hline Al 1 vs Al2 & 1 & 0,00 & 0,00 & 0,00 & 1,00 & ns \\
\hline Error & 10 & 8101,17 & 810,12 & & &
\end{tabular}

Table 7

Análisis de varianza de $t C \cdot h a^{-1}$ en la necromasa.

\begin{tabular}{l|l} 
F. Var & GI \\
\hline Total & 17 \\
\hline Bloques & 2 \\
\hline Tratamiento & 5 \\
\hline Altitud & 1 \\
Vegetación & 1 \\
\hline Interacción & 1 \\
\hline Ts vs Resto & 1 \\
\hline Al 1 vs Al2 & 1 \\
\hline Error & 10 \\
CV\% & \\
Media &
\end{tabular}

\begin{tabular}{l|l} 
S. Cuad & C. Medio \\
186,44 & \\
0,05 & 0,02 \\
184,74 & 36,95 \\
7,99 & 43,14 \\
126,92 & 126,92 \\
\hline 6,20 & 6,20 \\
\hline 43,14 & 43,14 \\
0,49 & 0,49 \\
1,65 & 0,17 \\
& 13,34 \\
& 3,05 \\
\hline
\end{tabular}

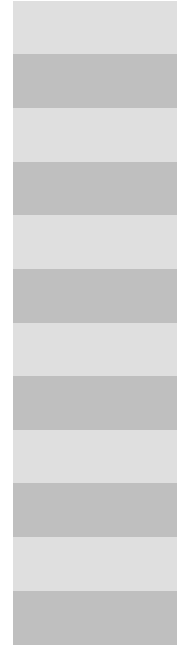

Fisher
0,14
223,33
260,74
767,16
37,45
260,74
2,98

\begin{tabular}{l|l} 
P. Fisher & Ínter. \\
\hline 0,87 & ns \\
0,00 & $* *$ \\
0,00 & $* *$ \\
0,00 & $* *$ \\
0,00 & $* *$ \\
0,00 & $* *$ \\
0,11 & ns \\
\hline & \\
\hline
\end{tabular}

de $0,89 \mathrm{tC} \cdot \mathrm{ha}^{-1}$ y $7,40 \mathrm{tC} \cdot \mathrm{ha}^{-1}$, respectivamente, y en el suelo a $20 \mathrm{~cm}$ de profundidad es de $111.84 \mathrm{tC} \cdot \mathrm{ha}^{-1}$ y $122.45 \mathrm{tC} \cdot \mathrm{ha}^{-1}$, respectivamente. En la zona de protección de paramo, se estimó que la cantidad de carbono almacenada es de 404738, 67 tC. El mismo que se encuentra distribuido de la siguiente manera: 149788.807 tC en 172,65 has de bosque nativo para la vegetación arbórea y 354949,86 tC en 1085,35 has de herbazal de páramo.

\section{References}

[1] CONDESAN (2013)

[2] GLORIA (Iniciativa para la Investigación y el Seguimiento Global de los Ambientes Alpinos) (2003).

[3] Rügnitz et al. (2009).

[4] IPCC (1996). 
[5] Calderon M, Lozada V. Determinación de biomasa y contenido de carbono en plantaciones forestales de Polylepis uncana y Polylepis reticulata [Determination of biomass and carbon content in forest plantations of Polylepis uncana and Polylepis reticulata,Tesis de grado del Internet]. Escuela Politécnica Nacional; 2010. Disponible en: http://www.sidalc.net/repdoc/A11216e/A11216e.pdf

[6] Pujos (2013).

[7] Londo (2018).

[8] MAE (2012).

[9] Smith Thomas M. Ecología [Ecology]. Madrid, España: S. R; 2007. Disponible en: http:www. geografiafisica.org/sem_2016_02/geo131/fuentes/SMITH-Ecologia.PDF. Spanish

[10] Ayala, Villa, Aguirre, \& Aguirre (2014).

[11] Santín y Vidal (2012).

[12] Mena P, Medina G. La biodiversidad de los páramos en el Ecuador [Biodiversity of the Paramos of Ecuador]; s/f. Disponible en: https://docplayer.es/6123304-La-biodiversidad-delos-paramos-en-el-ecu ador-patricio-mena-vasconez-y-galo-medinaintroduccion.html. Spanish

[13] Couteaux et al. (2002).

[14] Alvarado M, Gavilánez A. Línea base de los cinco humedales de la comunidad Chocavi-Páramo de Igualata [baseline of the five wetlands of the Chocavi-Paramo of the Igualata community,Tesis de grado del Internet]. Riobamba, Ecuador: Escuela Superior Politécnica de Chimborazo; 2013 [citado 2018 Nov 15]. Disponible en: file:///C:/Users/Usuario/Downloads/236T0177\%20(1).pdf. Spanish

[15] Arcos M. Influencia de la cobertura vegetal en la capacidad de inflitración de agua en suelos de páramo [influence of the vegetation cover on the capacity of water infiltration in Paramo soils,Tesis de pregrado del Internet]. Obtenido de Universidad San Francisco; 2010. Spanish

[16] Baca-G AE. Reflexiones sobre los procesos de ocupación humana en los páramos. Situación actual del páramo Volcán Chiles [Reflections on the processes of human occupation in the moors. Current situation of the Chiles Volcano Paramo,Internet]. Revista UDCA Actualidad \& Divulgación Científica. 2014;17:217-226. http://www.scielo.org.co/pdf/rudca/v17n1/v17n1a24.pdf. Spanish

[17] Bayas D. Diversidad florística a diferente altitud en el ecosistema páramo del Cantón Tisaleo Provincia de Tunguragua [flora diversity at different altitudes in the Paramo ecosystem of the Cantón Tisaleo Province of Tunguragua,Tesis de grado ingeniero forestal del Internet]. Riobamba: Escuela Superior Politécnica de Chimborazo; 2015. Disponible en: http://dspace.espoch.edu.ec/bitstream/123456789/ 3953/1/33T0142\{\%\}20.pdf. Spanish

[18] Caranqui J, Lozano P, Julio R. Composición y diversidad florística de los páramos en la Reserva e Producción de Fauna Chimborazo, Ecuador [composition and floristic diversity of the Paramos in the Chimborazo wildlife reserve and production, Ecuador,Internet]. Enfoque UTE. 2016;7(1). https://www.academia.edu/24022075/Composici\{\%\}C3\{\%\}B3n_y_diversidad_ flor\{\%\}C3\{\%\}ADstica_de_los_p\{\%\}C3\{\%\}A1ramos_en_la_Reserva_de_Producci\{\%\}C3\{\%\}B3n_ de_F auna_Chimborazo_Ecuador_Composition_and_diversity_of_High_Andean_in_the_ Fauna_Production_Reserve_Chimborazo_Ecuador. Spanish

[19] Casasbuenas L, Prieto J, Estupiñán LH. Efecto de la quema sobre algunas propiedades físico-químicas del suelo en el páramo Rabanal, Samacá Boyacá [effect of burning on some physical-chemical properties of the soil in the Rabanal Paramo, Samacá Boyacá]. Revista UDCA Actualidad \& Divulgación Científica. 2006;9(1):97-106. Spanish

[20] Cortés-Duque J, Sarmiento C. Visión socioecosistémica de los páramos y la alta montaña colombiana: memorias del proceso de definición de criterios para la delimitación de páramos [Socioecosystemic vision of the Paramos and the Colombian high mountains: memories of the process of defining criteria for the delimitation of Paramos,Internet]. 2013. Obtenido de: Instituto de Investigation de Recursos Biológicos Alexander von Humboldt. Disponible en: file://C:/Users/Xp/Downloads/vision_ socioecosistemica_paramos.pdf. Spanish

[21] Talleres de la Corporación de Estudios y Publicaciones [workshops of the Corporation of Studies and Publications]. Corporación de Estudios y Publicaciones. Ecuador. 2017. Legislación forestal. Spanish

[22] Food and Agriculture Organization. Captura de carbono en los suelos para un mejor manejo de la tierra [Capture of carbon in tthe ground for better land management,Internet]. Roma;2002. Disponible en: http://www.fao.org/3/a-bl001s.pdf. Spanish

[23] Food and Agriculture Organization. 2017. Inventario forestal. Obtenido de Conjunto de Herramientas para la Gestión Forestal Sostenible (GFS), Organización de las naciones unidas para la alimentación y agricultura: Disponible en: http://www.fao.org/sustainableforest-management/toolbox/modules/ forest-\{\%\}20inventory/basic-knowledge/es/.

[24] Haro F. 2012. Determinación de carbono orgánico en la biomasa y suelo del páramo de la comunidad Pichan Central-San Isidro [Determination of organic carbon en the biomass and the ground of the Paramo of the Pichan Central-San Isidro community,Tesis de grado del Internet]. Ecuador: Escuela 
Superior Politécnica de Chimborazo; 2012. Desponsible en: http://dspace.espoch.edu.ec/bitstream/ 123456789/2495/1/236T0076.pdf. Spanish

[25] Hofstede R, Mena P, Segarra P. Los páramos del mundo:proyeco atlas mundial de los Páramos [The paramos of the world:Global Paramo atlas project,Mapa]. EcoCiencia Fundación Ecuatoriana de estudios Ecológicos, EC. Comité Nacional de la UICN de los Países Bajos; 2003. Desponsible de: https://portals.iucn.org/library/node/8392. Spanish

[26] Lorenzo E. El mercado de carbono en América Latina y el Caribe: balance y perspectivas [The carbon market in Latin America and the Caribbean: balance and perspectives,Internet]. Santiago de Chile: Naciones Unidas; 2004 Mar. Disponible en: https://repositorio.cepal.org/bitstream/handle/11362/5620/ 1/S043136_es.pdf. Spanish.

[27] Loyola R. Guía de inventario de la flora y vegetación [Flora and vegetation inventory guide,Internet]. Ministerio del Ambiente; 2015. Disponible en: http://www.minam.gob.pe/patrimonio-natural/wpcontent/ uploads/sites/6/2013/10/GU\{\%\}C3\{\%]83-A-DE-FLORA-YVEGETACI\{\%\}C3\{\%\}83\{\%\}E2\{\%\}80\{\%\}9CN. compressed.pdf. Spanish

[28] Mayer C. Una mirada a los impactos del programa GESOREN en Tungurahua [A look at the impacts of the GESOREN program in Tungurahua,Inernet]; 2013. Disponible en: https://iiif. deutsche-digitale-bibliothek.de/binary/7ba5ae6f-92d8-4398-9c23-ea7f5715904f. Spanish

[29] Medina G. Paramo, El páramo como espacio de mitigación de carbono atmosférico [The Paramo as a space for mitigation of atmospheric carbon]. Órgano de difusión del grupo de trabajo en paramos del Ecuador: Editorial Abya Yala, Casilla 17-12-719; 1999. Spanish.

[30] Mena P, Hosfstede R. Los páramos del Ecuador [The paramos of Ecuador]. Obtenido de Proyecto de Páramo. 2006. Disponible en:: http://biblio.flacsoandes.edu.ec/catalog/resGet.php?resld=49243. Spanish

[31] Mota Cesar C. Investigación sobre la absorción de CO2 por los cultivos más representativos [Research on the absorption of $\mathrm{CO} 2$ by the most representative crops,Internet]. Less CO2: A.-L.-B; s/f. Disponible en: http://www.lessco2.es/pdfs/noticias/ponencia_cisc_espanol.pdf. Spanish

[32] Padilla E. Análisis de las emisiones de $\mathrm{CO} 2$ y sus factores explicativos en las diferentes áreas de mundo [Analysis of $\mathrm{CO} 2$ emissions and their explanatory factors in different areas of the world,Internet]. Research Gate; 2005. Diesponsibe en: https://www.researchgate.net/publication/24138217_Analisis_ de_las_emisiones_de_CO2_y_sus_factores_explicativos_en_las_diferentes_areas_del_mundo. Spanish

[33] Podwojewski P, Poulenard J. La degradación de los suelos de los páramos [The degredation of the soild of the Paramos]. Serie solos del paramo. Serie Paramo. 2000;5:27-36. Spanish

[34] Los suelos andinos y sus componentes [Andean soils and their components]. PROMAS Programa de manejo de agua y suelo. Cuenca, Ecuador. 2009. Spanish

[35] Ramírez M. Diversida floristica a diferente altitud en el ecosistema páramo en siete comunidades de OSG UNOCANT [Floristic diversity at different altitudes in the Paramo ecosystem in seven communities of OSG UNOCANT,Tesis ingenieria forestal del Internet]. Riobamba: Escuela Superior Politécnica de Chimborazo; 2013. Desponsible de: http://dspace.espoch.edu.ec/bitstream/123456789/ 2790/1/33T0112[\%]20.pdf. Spanish

[36] Sturm H, Rangel O. Ecología de los páramos andinos: Una visión preliminar integrada [Ecology of the Andean Paramos: An integrated preliminary view]. Bogotá: Instituto de Ciencias Naturales ICN, Universidad Nacional de Colombia; 1985. 292p. Spanish 